\title{
Self-Determination Theory and First-Episode Psychosis: A Replication
}

\author{
Nicholas J. K. Breitborde, ${ }^{1}$ Cindy Woolverton, ${ }^{1}$ R. Brock Frost, ${ }^{2}$ and Nicole A. Kiewel ${ }^{3}$ \\ ${ }^{1}$ Department of Psychiatry, The University of Arizona, Tucson, AZ 85713, USA \\ ${ }^{2}$ Department of Psychiatry, University of New Mexico, Albuquerque, NM 87131, USA \\ ${ }^{3}$ Department of Psychiatry, Cleveland Clinic, Cleveland, OH 44195, USA
}

Correspondence should be addressed to Nicholas J. K. Breitborde; breitbor@email.arizona.edu

Received 24 June 2014; Accepted 15 August 2014; Published 8 September 2014

Academic Editor: Takahiro Nemoto

Copyright (C) 2014 Nicholas J. K. Breitborde et al. This is an open access article distributed under the Creative Commons Attribution License, which permits unrestricted use, distribution, and reproduction in any medium, provided the original work is properly cited.

\begin{abstract}
Self-determination theory (SDT) posits that human well-being depends on the satisfaction of three basic psychological needs: autonomy, competence, and relatedness. Although many scholars have suggested that SDT may be relevant to psychotic disorders, only one empirical study of SDT in individuals with psychosis has been completed to date by Breitborde and colleagues (2012). This study revealed that individuals with first-episode psychosis reported lower satisfaction of the three basic psychological needs as compared to individuals without psychosis. Moreover, greater satisfaction of basic psychological needs was modestly associated with lower general symptoms (e.g., anxiety and depression), greater social functioning, and better quality of life. Thus, the goal of this project was to replicate Breitborde et al.s (2012) investigation of basic psychological need satisfaction among individuals with first-episode psychosis. Our results supported the conclusion that individuals with first-episode psychosis report lower autonomy, competence, and relatedness than individuals without psychosis. Moreover, our results comport with the finding that greater need satisfaction was associated with less severe symptomatology and better social functioning and quality of life. In total, the findings lend further credence to the hypothesis that SDT may help to inform the development of improved clinical services for individuals with psychotic disorders.
\end{abstract}

\section{Introduction}

Self-determination theory (SDT: [1]) posits that human wellbeing and motivation are dependent on the satisfaction of three basic psychological needs: autonomy (i.e., viewing oneself as the volitional source of one's actions), competence (i.e., perceiving one's self as effective in interactions in one's local world), and relatedness (i.e., feeling a sense of connectedness and belongingness with others in one's local world). Recently, several scholars have suggested that SDT theory may be relevant to psychotic disorders $[2,3]$. For example, Choi and colleagues [4] have shown that greater intrinsic motivationa construct underpinned by the three basic psychological needs [1] - is associated with greater participation in and response to cognitive remediation among individuals with schizophrenia.

Yet, despite this potential utility of SDT, only one study to date has examined basic psychological need satisfaction among individuals with psychosis. In this study, Breitborde and colleagues [5] found that individuals with first-episode psychosis reported lower feelings of autonomy, competence, and relatedness as compared to individuals without psychosis. Moreover, greater satisfaction of basic psychological needs was modestly associated with lower general symptoms (e.g., anxiety and depression), greater social functioning, and better quality of life. Among the three basic psychological needs, relatedness was found to be the most frequent correlate of clinical and functional status among individuals with firstepisode psychosis.

With only one investigation of basic psychological need satisfaction among individuals with psychosis completed to date, the reliability of Breitborde and colleagues' [5] findings is unclear. This limitation is not unique to the Breitborde et al. study specifically; within psychiatric research, there is growing recognition of the limited reliability of findings from single studies $[6,7]$. In response to this concern, scholars have 
advocated for the systematic replication of research findings prior to assuming that such findings are reliable $[8,9]$.

Thus, the goal of this project is to replicate Breitborde and colleagues' [5] investigation of basic psychological need satisfaction among individuals with first-episode psychosis. Consistent with the previous study, we first compared levels of basic psychological need satisfaction among individuals with first-episode psychosis to levels of need satisfaction among individuals without psychosis. Second, we investigated the association between need satisfaction and measures of symptomatology, social functioning, and quality of life among individuals with first-episode psychosis.

\section{Methods}

2.1. Participants. Forty-three individuals with first-episode psychosis were recruited from the Early Psychosis Intervention Center (EPICENTER: [10]). EPICENTER eligibility criteria include (i) diagnosis of a schizophrenia-spectrum or affective disorder with psychotic features as determined using the Structured Clinical Interview for the DSM-IV [11], (ii) less than 5 years of psychotic symptoms established using the Symptom Onset in Schizophrenia Inventory [12], (iii) ages 18-35, and (iv) no evidence of mental retardation or organic brain impairment. Participants in the study include 31 males and 12 females with an average age of 22.16 years $(\mathrm{SD}=4.10)$. The median duration of psychotic symptoms was 13.94 months.

This project was completed in accordance with the Declaration of Helsinki. The study was approved by the University of Arizona Institutional Review Board, and all participants provided informed consent prior to enrolling in this study.

\subsection{Procedures and Measures. Upon enrollment in EPICEN-} TER and prior to the start of treatment, participants completed a series of assessments that were part of a larger research battery.

2.3. Basic Psychological Needs. Basic psychological need satisfaction was assessed using the Basic Psychological Needs Scale-General (BPNS: [13]). The BPNS is a 21-item selfreport measure that assesses satisfaction of the need for autonomy, competence, and relatedness across life in general. All items are scored on seven-point Likert scale, with higher scores indicative of greater need satisfaction. Psychometric evaluations of the BPNS have revealed that this measure possesses good to excellent internal consistency $[13,14]$. The BPNS also has demonstrated predictive validity with regard to differentiating between individuals with psychopathology versus those without $[5,14]$.

Normative data for the BPNS are lacking. However, in their previous study, Breitborde and colleagues [5] created pseudonormative data for the three BPNS subscales (i.e., autonomy, competence, and relatedness) from the 9145 individuals without a known psychotic disorders included in all previously published studies that used the 17-item BPNS and reported means and standard deviations. More specifically, they calculated means and standard deviations for each BPNS subscale weighted by the number of subjects in each study. A list of these studies can be found in Breitborde et al. [5]. Among the 9145 individuals in these studies, the average age was 20.45 years $(\mathrm{SD}=2.98)$ and $39 \%$ of the individuals were male.

2.4. Symptomatology. The Positive and Negative Syndrome Scale (PANSS: [15]) was used to assess symptomatology among study participants. The PANSS is a 30 -item clinicianrated instrument that assesses three domains of symptomatology: positive symptoms (e.g., hallucinations and delusions), negative symptoms (e.g., blunted affect and social withdrawal), and general symptoms (e.g., anxiety and depression). Higher PANSS scores are indicative of worse symptomatology.

2.5. Social Functioning. Participants' level of social functioning was measured using the Social Functioning Scale (SFS: [16]). The SFS is a 79-item instrument that assesses seven areas of functioning: (i) social engagement/withdrawal, (ii) interpersonal behavior/communication, (iii) participation in prosocial activities, (iv) participation in recreational activities, (v) independence competence (i.e., ability to perform tasks of independent living), (vi) independence performance (i.e., completion of tasks of independent living), and (vii) educational/vocational functioning. Higher scores on the SFS are indicative of greater social functioning.

2.6. Quality of Life. The WHO Quality of Life InstrumentBrief (WHOQOL-BREF: [17]) was used to assess quality of life among study participants. The WHOQOL-BREF is a 26item self-report scale that measures four domains of quality of life: (i) physical health, (ii) psychological health, (iii) social relationships, and (iv) quality of one's environment. Scores for each domain range from 0 to 100 , with higher scores indicative of greater quality of life.

2.7. Analyses. Prior to the analyses, missing data points were replaced using multiple imputation (MI: [18]). This strategy comports with current guidelines for addressing missing data [19]. Comparison of BPNS scores between individuals with first-episode psychosis and individuals without a known psychotic disorder was completed using independent $t$ tests controlling for the additional variance created through the use of MI [20]. Evaluation of the association between BPNS subscales and measures of symptomatology, social functioning, and quality of life was completed using Pearson correlation coefficients controlling for the additional variance created through the use of MI [20].

\section{Results}

3.1. Satisfaction of Basic Psychological Needs. Among individuals with first-episode psychosis, there were no statistically significant differences between scores on the autonomy, competence, and relatedness subscales (all $P$ values $>0.05$ ). BPNS scores for individuals with first-episode psychosis versus those with no known psychotic disorder are shown 
TABLE 1: Demographics and BPNS scores among individuals with first-episode psychosis versus individuals with no known psychotic disorder.

\begin{tabular}{lcc}
\hline & $\begin{array}{c}\text { Individuals with } \\
\text { first-episode } \\
\text { psychosis }\end{array}$ & $\begin{array}{c}\text { Individuals with no } \\
\text { known psychotic } \\
\text { disorder }\end{array}$ \\
\hline$N$ & 43 & 9145 \\
Age & $\mathrm{M}=22.16$ & $\mathrm{M}=20.45$ \\
Percent male-female & $74 \%-26 \%$ & $39 \%-61 \%$ \\
Autonomy & $\mathrm{M}=4.26$ & $\mathrm{M}=5.00$ \\
Competence & $\mathrm{M}=4.04$ & $\mathrm{M}=5.07$ \\
Relatedness & $\mathrm{M}=4.18$ & $\mathrm{M}=5.33$ \\
\hline
\end{tabular}

in Table 1. Compared to individuals without psychosis, individuals with first-episode psychosis reported lower autonomy $[t(7622)=5.43 ; P<0.01]$, competence $[t(7924)=7.15 ; P<$ $0.01]$, and relatedness $[t(7945)=8.26 ; P<0.01]$.

Of note, there were a number of demographic variables that differed between the individuals with first-episode psychosis and the participants from the comparison studies (see Table 1). Specifically, individuals with first-episode psychosis were more likely to be men $\left[\chi^{2}(1)=19.93 ; P<0.01\right]$ and were older $[t(8320)=3.74 ; P<0.01]$ than the individuals without psychosis. Available research suggests that there is no association between gender and psychological need satisfaction [21]. Moreover, although need satisfaction has been shown to increase with age [22], our sample of individuals with first-episode psychosis reported lower need satisfaction than the younger cohort of individuals without a known psychotic disorder.

3.2. Symptomatology. Correlations between BPNS and PANSS are shown in Table 2 . There were no statistically significant associations between severity of positive or negative symptoms and levels of autonomy, competence, or relatedness. Higher levels of general symptoms were associated with lower levels of autonomy and competence.

3.3. Social Functioning. Correlations between BPNS and SFS are shown in Table 2. Better interpersonal communication was associated with greater autonomy, competence, and relatedness. Higher levels of competence were also associated with greater success in the performance of independent living skills (i.e., Independence-Performance subscale). Greater perceived competence with regard to the performance of independent living skills (i.e., Independence-Competence subscale) was associated with higher autonomy and competence.

3.4. Quality of Life. Correlations between BPNS and WHOQOL-BREF are shown in Table 2. Better physical health was associated with higher levels of autonomy. Greater psychological health was associated with higher levels of autonomy, competence, and relatedness. Better social relationships were associated with greater autonomy and relatedness. There were no statistically significant associations between quality of one's environment and BPNS subscales.

\section{Discussion}

Consistent with the results from Breitborde et al. [5], individuals with first-episode psychosis in our study reported lower autonomy, competence, and relatedness than individuals without psychosis. Similar reports of reduced need satisfaction have also been noted in studies of individuals with other forms of psychopathology (e.g., posttraumatic stress disorder [14] and major depressive disorder [23]). Our finding likely cannot be accounted for due to demographic differences between the two groups (i.e., gender and age). Available research suggests that there is no association between gender and psychological need satisfaction [21]. Moreover, although need satisfaction has been shown to increase with age [22], our sample of individuals with first-episode psychosis reported lower need satisfaction than the younger cohort of individuals without a known psychotic disorder. Additionally, our results further comport with findings by Breitborde et al. in that we found modest associations between basic psychological need satisfaction and symptomatology, social functioning, and quality of life among individuals with firstepisode psychosis.

Of note, there is a key difference between our results and those reported by Breitborde and colleagues [5]. Specifically, whereas Breitborde et al. found that relatedness was the psychological need most frequently associated with clinical/functional status, among our sample, no single psychological need stood out as the best predictor of clinical/functional status. Important differences among the participants in these two studies may have contributed to this finding. For example, compared to participants in the Breitborde et al. study, our subjects had a longer duration of psychotic symptoms (Median $=13.94$ versus 3.46 months, Wilcoxon rank-sum $z=-3.71 ; P<0.01)$ but a shorter duration of participation in specialized treatment for first-episode psychosis (Mean $=0$ versus 9.91 months, Wilcoxon rank-sum $z=5.22 ; P<0.01)$. This raises the possibility that satisfaction of the needs for autonomy and competence may be more important later in the early course of psychotic disorders and/or earlier during individuals' participation in specialized clinical services for first-episode psychosis. Relatedness, on the other hand, may be important throughout the early course of psychotic disorders as well as throughout participation in care for first-episode psychosis.

Our study did suffer from a number of limitations. The sample size was small and comprised predominantly of men. Likewise, our results may not generalize to individuals with longstanding psychotic illnesses. Finally, given our cross-sectional study design, the direction of the relationship between psychological need satisfaction and clini$\mathrm{cal} /$ functional status is unclear.

In conclusion, the results of our replication study support the hypothesis that deficits in satisfaction of basic psychological needs are present early in the course of psychotic disorders and are associated with measures of clinical/functional status. 
TABLE 2: Symptomatology, social functioning, and quality of life: descriptive statistics and correlations with BPNS scores.

\begin{tabular}{|c|c|c|c|c|}
\hline & $\mathrm{M}(\mathrm{SD})$ & Autonomy & Competence & Relatedness \\
\hline \multicolumn{5}{|l|}{ Symptomatology } \\
\hline Positive symptoms & $15.30(2.41)$ & -0.16 & -0.22 & -0.09 \\
\hline Negative symptoms & $13.71(2.33)$ & 0.12 & 0.13 & -0.02 \\
\hline General symptoms & $30.95(2.66)$ & $-0.41^{*}$ & $-0.36^{*}$ & -0.25 \\
\hline \multicolumn{5}{|l|}{ Social functioning } \\
\hline Social engagement/withdrawal & $10.24(1.74)$ & 0.15 & 0.29 & 0.20 \\
\hline Interpersonal communication & $6.86(1.55)$ & $0.42^{*}$ & $0.50^{*}$ & $0.44^{*}$ \\
\hline Independence performance & $23.60(2.77)$ & 0.27 & $0.42^{*}$ & 0.18 \\
\hline Recreation & $17.72(2.58)$ & 0.19 & 0.12 & -0.03 \\
\hline Prosocial activities & $14.35(2.95)$ & 0.13 & 0.24 & 0.06 \\
\hline Independence competence & $34.13(2.35)$ & $0.36^{*}$ & $0.35^{*}$ & 0.20 \\
\hline Educational/occupational functioning & $6.45(1.90)$ & 0.13 & 0.10 & 0.06 \\
\hline \multicolumn{5}{|l|}{ Quality of life } \\
\hline Physical health & $53.16(5.43)$ & $0.40^{*}$ & 0.19 & 0.16 \\
\hline Psychological health & $39.65(5.48)$ & $0.48^{*}$ & $0.40^{*}$ & $0.36^{*}$ \\
\hline Social relationships & $34.31(5.21)$ & $0.43^{*}$ & 0.18 & $0.35^{*}$ \\
\hline Environment & $52.63(5.44)$ & 0.29 & 0.21 & 0.10 \\
\hline
\end{tabular}

Ultimately, developing clinical environments designed to address these deficits may facilitate greater motivation among individuals with first-episode psychosis to participate in treatment activities $[2,3]$.

\section{Conflict of Interests}

The authors declare that there is no conflict of interests regarding the publication of this paper.

\section{Acknowledgments}

This work was supported by funds from the University of Arizona and grant from the Institute for Mental Health Research to Nicholas J. K. Breitborde.

\section{References}

[1] R. M. Ryan and E. L. Deci, "Self-determination theory and the facilitation of intrinsic motivation, social development, and well-being," The American Psychologist, vol. 55, no. 1, pp. 68-78, 2000.

[2] A. D. Mancini, "Self-determination theory: a framework for the recovery paradigm," Advances in Psychiatric Treatment, vol. 14, no. 5, pp. 358-365, 2008.

[3] A. Medalia and J. Brekke, "In search of a theoretical structure for understanding motivation in schizophrenia," Schizophrenia Bulletin, vol. 36, no. 5, pp. 912-918, 2010.

[4] J. Choi, T. Mogami, and A. Medalia, "Intrinsic motivation inventory: an adapted measure for schizophrenia research," Schizophrenia Bulletin, vol. 36, no. 5, pp. 966-976, 2010.

[5] N. J. K. Breitborde, P. Kleinlein, and V. H. Srihari, "Selfdetermination and first-episode psychosis: associations with symptomatology, social and vocational functioning, and quality of life," Schizophrenia Research, vol. 137, no. 1-3, pp. 132-136, 2012.

[6] J. P. Ioannidis, "Why most published research findings are false," PLoS Medicine, vol. 2, no. 8, p. e124, 2005.

[7] Y. Amir and I. Sharon, "Replication research: a " must" for the scientific advancement of psychology," Journal of Social Behavior \& Personality, vol. 5, no. 4, pp. 51-69, 1990.

[8] R. Moonesinghe, M. J. Khoury, and A. C. J. W. Janssens, "Most published research findings are false-but a little replication goes a long way," PLoS Medicine, vol. 4, no. 2, p. e28, 2007.

[9] J. Cohen, “The earth is round ( $<$ 0.05)," The American Psychologist, vol. 49, no. 12, pp. 997-1003, 1994.

[10] N. J. K. Breitborde et al., "The Early Psychosis Intervention Center: exploring the mechanisms of change for psychosocial interventions for first-episode psychosis," Early Intervention in Psychiatry, vol. 4, supplement 1, p. 56, 2010.

[11] M. B. First, R. L. Spitzer, M. Gibbon, and J. B. W. Williams, Structured Clinical Interview for DSM-IV-TR Axis I Disorders, Research Version, Patient Edition (SCID-I/P), Biometrics Research, New York State Psychiatric Institute, New York, NY, USA, 2002.

[12] D. O. Perkins, J. Leserman, L. F. Jarskog, K. Graham, J. Kazmer, and J. A. Lieberman, "Characterizing and dating the onset of symptoms in psychotic illness: the Symptom Onset in Schizophrenia (SOS) inventory," Schizophrenia Research, vol. 44, no. 1, pp. 1-10, 2000.

[13] M. Gagné, "The role of autonomy support and autonomy orientation in prosocial behavior engagement," Motivation and Emotion, vol. 27, no. 3, pp. 199-223, 2003.

[14] T. B. Kashdan, T. Julian, K. Merritt, and G. Uswatte, "Social anxiety and posttraumatic stress in combat veterans: relations to well-being and character strengths," Behaviour Research and Therapy, vol. 44, no. 4, pp. 561-583, 2006.

[15] S. R. Kay, A. Fiszbein, and L. A. Opler, "The positive and negative syndrome scale (PANSS) for schizophrenia," Schizophrenia Bulletin, vol. 13, no. 2, pp. 261-276, 1987. 
[16] M. Birchwood, J. Smith, R. Cochrane, S. Wetton, and S. Copestake, "The Social Functioning Scale. The development and validation of a new scale of social adjustment for use in family intervention programmes with schizophrenic patients," The British Journal of Psychiatry, vol. 157, pp. 853-859, 1990.

[17] The WHOQOL Group, "Development of the World Health Organization WHOQOL-BREF quality of life assessment," Psychological Medicine, vol. 28, no. 3, pp. 551-558, 1998.

[18] D. B. Rubin, Multiple Imputation for Non-Response in Surveys, John Wiley \& Sons, New York, NY, USA, 1987.

[19] J. W. Graham, "Missing data analysis: making it work in the real world," Annual Review of Psychology, vol. 60, pp. 549-576, 2009.

[20] P. Royston, "Multiple imputation of missing values: update of ice," Stata Journal, vol. 5, no. 4, pp. 527-536, 2005.

[21] T. B. Kashdan, A. Mishra, W. E. Breen, and J. J. Froh, "Gender differences in gratitude: examining appraisals, narratives, the willingness to express emotions, and changes in psychological needs," Journal of Personality, vol. 77, no. 3, pp. 691-730, 2009.

[22] J. A. Hicks, J. Trent, W. E. Davis, and L. A. King, "Positive affect, meaning in life, and future time perspective: an application of socioemotional selectivity theory," Psychology and Aging, vol. 27, no. 1, pp. 181-189, 2012.

[23] M. S. Ibarra-Rovillard and N. A. Kuiper, "Social support and social negativity findings in depression: perceived responsiveness to basic psychological needs," Clinical Psychology Review, vol. 31, no. 3, pp. 342-352, 2011. 


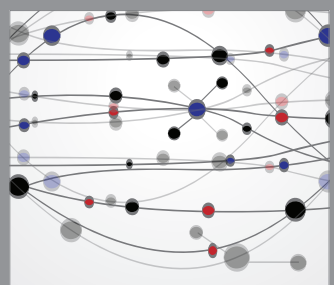

The Scientific World Journal
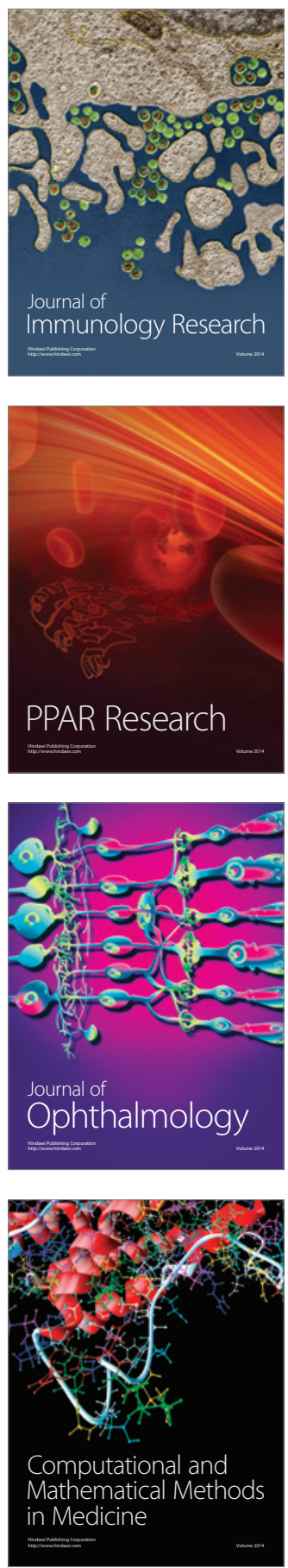

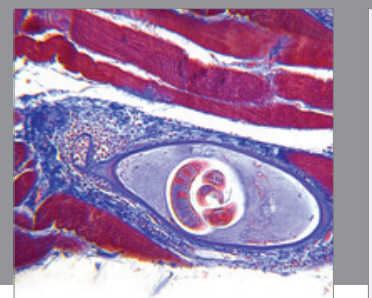

Gastroenterology

Research and Practice
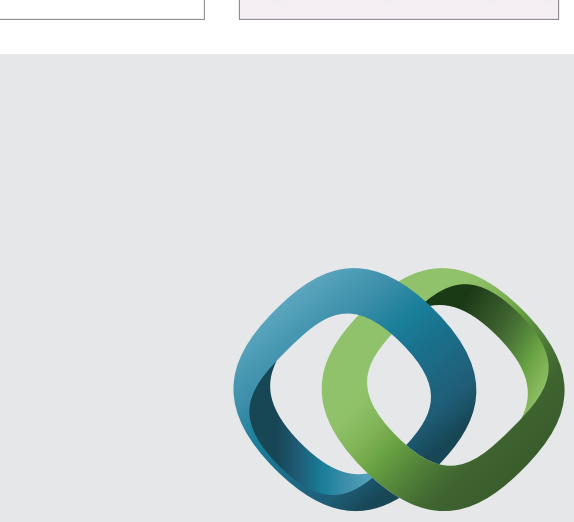

\section{Hindawi}

Submit your manuscripts at

http://www.hindawi.com
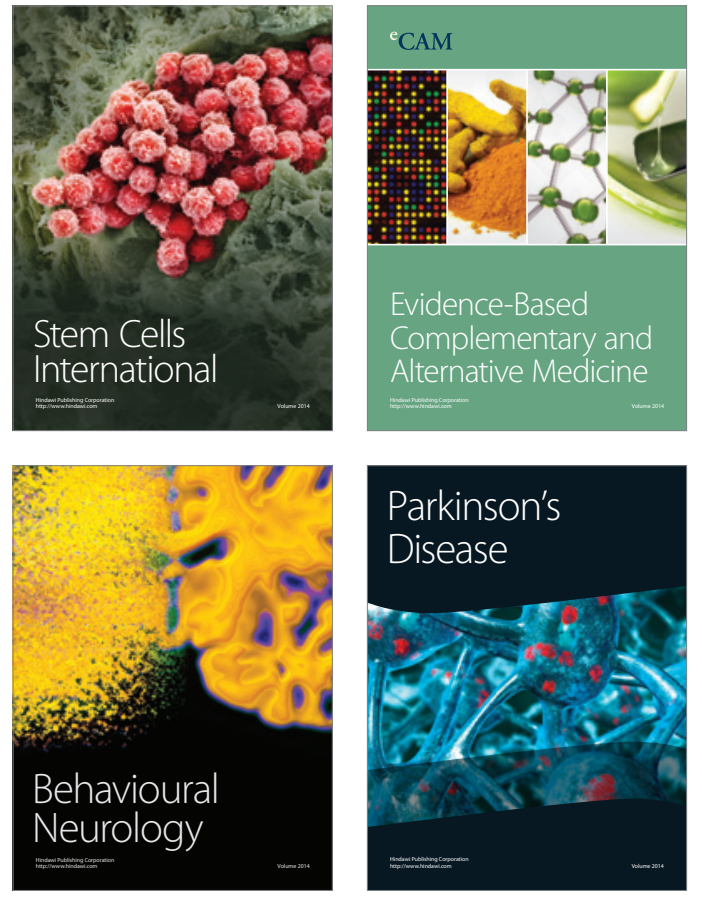
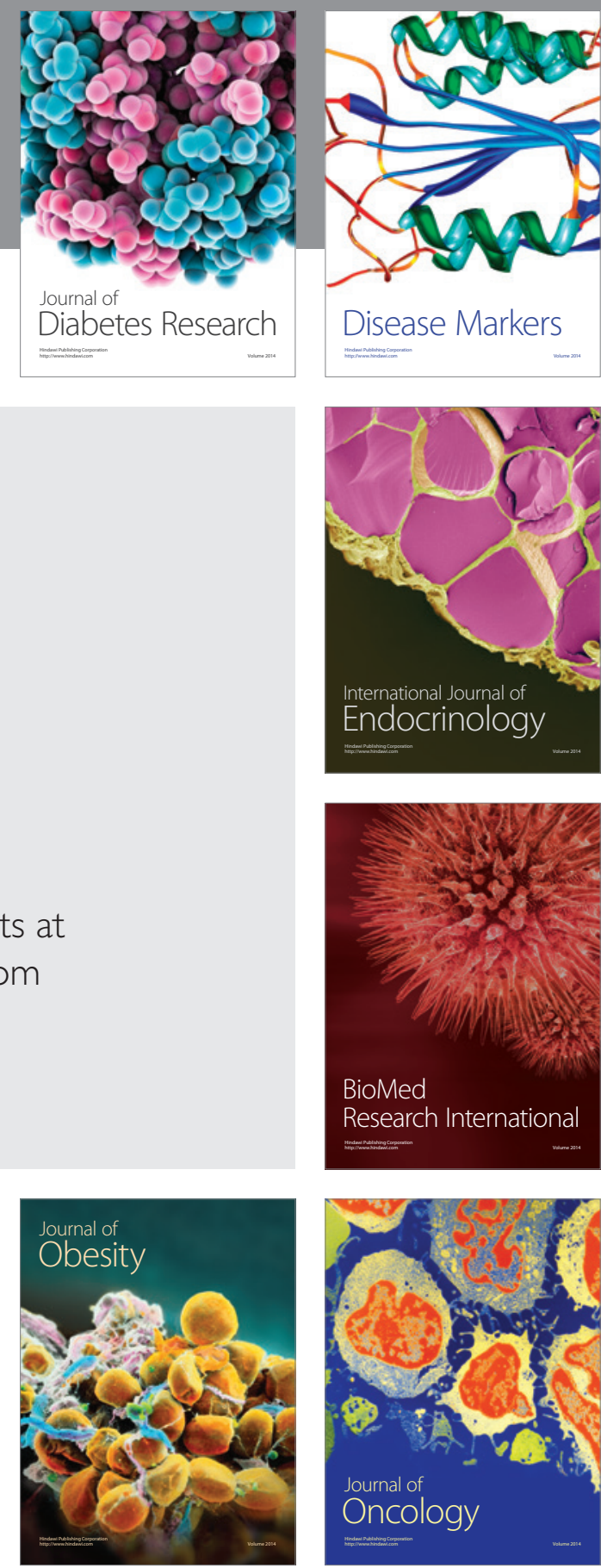

Disease Markers
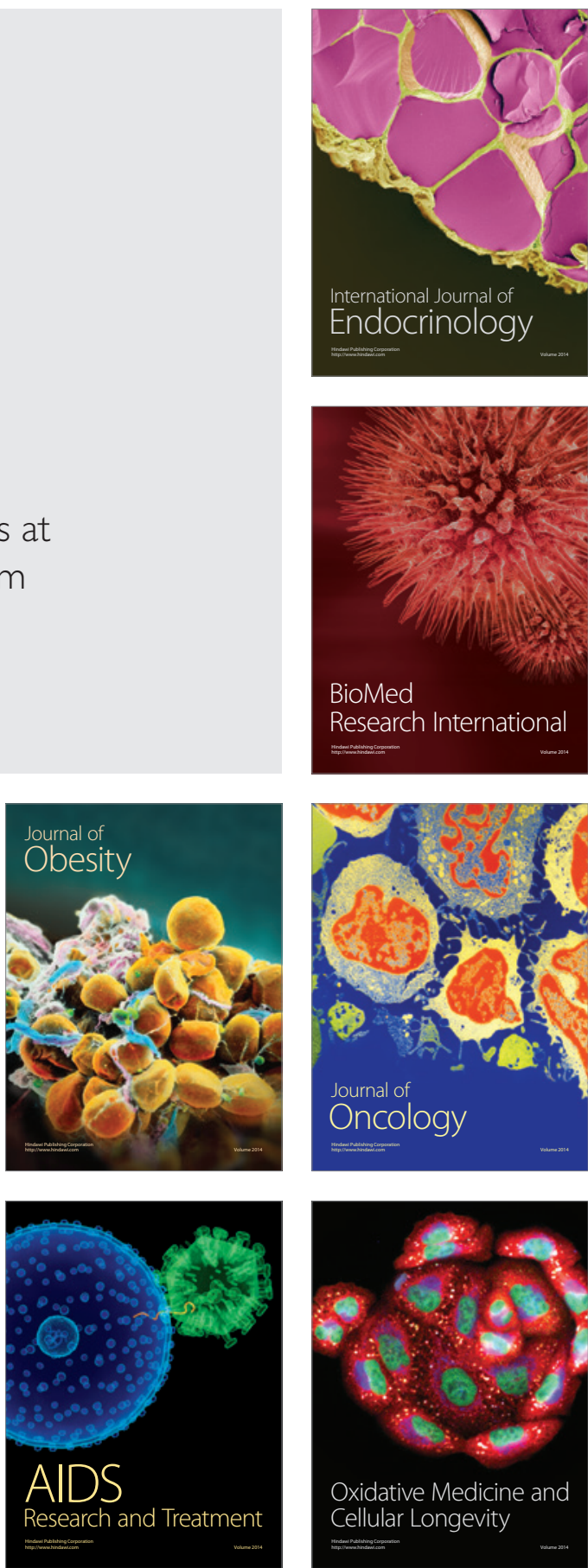